\section{Psychological Perceptions and Preparedness during Novel Coronavirus Disease - 2019 (COVID-19) Pandemic - A Multinational Cross-sectional Study among Healthcare Professionals}

Arishiya Thapasum Fairozekhan(D1, Shamaz Mohamed(D2, Faraz Mohammed(D1, Ramesh Kumaresan(D3, Amr Saeed Mabark Bugshan(1), Sudeep C. BD4, Shakil Moidin 5, Saud Abdullah AIMoumen (D6.

Novel Coronavirus Disease 2019 (COVID-19) has caused serious repercussions both physically and mentally. The crisis has laid an enormous workload on the global healthcare fraternity. This article has attempted to study the emotional and psychological status of the medical and dental fraternity across seven Asian countries (India, Malaysia, the Gulf Cooperation countries (GCC) and, others) and also deduce the degree of mental preparedness as they spearhead the war against COVID-19. A cross-sectional, descriptive online survey was carried out among potential participants from online forums and other health-related social communities. Bivariate analysis with descriptive statistics was applied to decipher the results. A total of 788 complete responses were analyzed. The response rate was $77.1 \%$. Results revealed the perception of the Healthcare Professionals about COVID-19 and its implications in their personal and professional lives. Many categories from psychological and emotional standpoints were analyzed. Older HCPs (above 35 years) reported high levels of stress at the workplace $(p=0.002)$. About $43.5 \%$ of the HCPs from India reported that they have not received any specialized training on containing COVID-19 or any contagious disease. Intense emotional stress was reported by the HCPs when colleagues get infected. The medical professionals (61.7\%) exhibited more work stress compared to their dental counterparts. Analyzing the psychological and emotional status of HCPs is imperative especially in this COVID-19 situation. Similar analyses are crucial to gauge the quality of our healthcare system and take necessary actions like training the workforce, revamping the infrastructure, and regulating the workflow.
1. Department of Biomedical Dental Sciences, College of Dentistry, Imam Abdulrahman Bin Faisal University, Dammam, Kingdom of Saudi Arabia.

2. Senior Drug Safety Physician-II, Indegene Private Limited (formerly, Indegene Lifesystems Private Limited), Manyata Tech Park, Bangalore, Karnataka, India.

\footnotetext{
3. Department of Oral \& Maxillofacial Surgery, Faculty of Dentistry, AIMST University, Darul Aman, Kedah, Malaysia.

4. Department of Community and Public Health Dentistry, Sree Anjaneya Institute of Dental Sciences, Malabar Medical College \& Hospital Campus, Calicut, Kerala, India.

5. Department of Maxillofacial Surgery and Diagnostic Sciences, College of Dentistry in AlRass, Qassim University, Al-Rass, Kingdom of Saudi Arabia.

6. Ministry of Health, Shoabat Nusab General Hospital, Northern Borders Province, Kingdom of Saudi Arabia

Correspondence: Dr. Faraz Mohammed Department of Biomedical Dental Sciences, College of Dentistry, Imam Abdulrahman Bin Faisal University, Dammam, Kingdom of Saudi Arabia.E-mail-fmkasti@iau.edu.sa jogalkasti@yahoo.co.in
}

Key Words: Perceptions, Preparedness, Stress, Readiness, Psychological concerns, COVID19, Coronavirus, Health care workers, Dentists in COVID-19 


\section{Introduction}

The recent pandemic has laid deplorable burdens on all people irrespective of age, race and occupation. Medical profession entails high sense of duty during contingencies. The global healthcare workforce has been greatly reinforced to fortify and strengthen herd immunity. Every HCP is obligated to patient safety, but there is no visible demarcation between a HCP's duty of care and personal protection during any pandemic, as COVID-19 (1). In fact, these obligations are greatly challenged during a pandemic. Many a report has revealed indecisive mental disposition of clinicians and healthcare professionals during pandemics (2). It is been reported that there will be chances of high vulnerability of burnout and extreme emotional exhaustion among HCPs specially in frontline workers based in China and Hong Kong $(3,4)$. As best to our knowledge, similar reports with HCPs working in Asian countries are not available other than China. Hence, we have attempted to study the emotional and psychological status of HCPs working in Asian countries with reference to the general healthcare setup in these countries and the real time challenges they encounter. It has been documented that there is a high prevalence of anxiety and work-related stress among the female HCPs compared to the male population (5). Although we did not analyze any gender-based differences, we dichotomized the respondents into two age groups ( $>35$ and $<35$ years) to understand if age and professional experience can intensify stress feelings.

As the viral infection disseminates like wildfire, more forefront workers are the need of the hour. The service of the dental community in this regard is greatly appreciated. Many countries are utilizing their dental fraternity for emergency COVID-19 duties. Although the scope of work is divergent, the dental population has been trained in basic medical practice and sterilized surgical techniques. Moreover, the dental practitioner has been trained to face medical emergencies in dental practice including immune-compromised patients (6). During this COVID-19 situation, dental practice has been greatly challenged, as the probability of disease dissemination is high during treatment. Therefore, the Centers for Disease Control and Prevention (CDC) and World Health Organization (WHO) have laid restrictive guidelines on elective dental treatments and in this regard, the dentist's workload has been greatly reduced. This available professional manpower can be greatly utilized during COVID-19. The National Health Scheme (NHS) has directed the dental fraternity to assist their medical counterparts to fight against COVID-19 and serve in emergency wards (7). These redeployed dental professionals fill in for the mainstream medical fraternity. In addition, many dental hospitals have now been rejuvenated as COVID19 care medical centers (8).

Healthcare institutions are expected to be key players during a pandemic, with healthcare workers at a high risk of exposure and infection. During the last epidemic of Severe Acute Respiratory Syndrome (SARS), and Middle East Respiratory Syndrome (MERS), healthcare workers suffered from significant stress, partly from an overstretched healthcare system (9). This is expected to be a similar scenario with the Novel Coronavirus Disease-2019 (COVID-19) pandemic. More than half of all secondary cases of COVID-19 confirmed by the laboratories were linked to healthcare settings (10). The Kingdom of Saudi Arabia's Ministry of Health on March 21, 2020 reported the transmission of COVID-19 to the healthcare workers in Saudi Arabia (11).

The present study focuses to explore and compare the various perceptions and preparedness for a COVID-19 pandemic between healthcare workers working in different countries. The objectives of the study were to identify factors that influence behaviour, safety measures at work, pandemic preparedness, responsibility for dependents, influences of personal life in discharging their duties at the workplace and further to correlate the above objective between dental and medical health care workforce.

\section{Materials and methods}

We conducted a cross-sectional, descriptive survey among HCPs belonging to the seven Asian countries viz. India, Malaysia, Gulf Cooperation Council (Kingdom of Saudi Arabia, Qatar, Oman, United Arab Emirates, and Kuwait). Further, HCPs who were on official deputations to certain countries (Singapore, Indonesia, United States of America, and Egypt) from the above seven countries were designated as respondents from other countries. The survey was conducted between April 15 to May 5, 2020. During this time, the pandemic had already permeated into every country and was rampant in Asian countries. This analysis was primarily taken to peruse the mental and psychological readiness of the medical and dental communities in Asia during the pandemic. The respondents of this questionnaire were frontline workers in COVID-19 wards and belonged to two distinctive groups viz. a) mainstream HCPs like physicians, specialists, medical students, nurses and; b) dental HCPs like dentists, dental 
assistants and dental students. The survey questionnaire was pretested for face validity. With limited sample of health workers, the questionnaire was standardized with Chronbach's alpha coefficient score (0.79). These samples were excluded from the final survey data. The data was collected at a phase when the dental fraternity was assisting the mainstream medical fraternity. The questionnaire was prepared in English and disseminated through social online medical/dental communities and forums. Both convenience sampling (researchers themselves contacted HCPs to participate in the study) and snowball sampling (the participating HCPs were asked to forward the questionnaire to their colleagues) were used so that maximal participation could be ensured.

\section{Data collection}

The questionnaire used in the survey was adapted from an early statistical study that surveyed the concerns and preparedness among health care workers during the Severe Acute Respiratory Syndrome (SARS) breakout (12).

The survey questionnaire consisted of 21 closed-ended questions using a "likelihood Likert scale". The scoring system for favorable questions is based on the following criteria: 'Yes', 'Somewhat', and 'No'.

The questionnaire assessed the impact of Perceptions and Preparedness among the healthcare workers during a pandemic - Pandemic Work/job-related concerns (10 items), stress perception related (6 items), and preparedness at the workplace (5 items). A "Questionpro" (Link_masked for review) link was used to disseminate the survey multinationally. The online survey was made available for a timeframe of 20 days. One of the limitations of this study could be attributed to the fact that there was no sampling frame as the questionnaires were distributed online; hence could be considered as both convenience and snowball sampling. However, given the study being multi-national and due to the prevailing pandemic situation, online survey was the best possible method for data collection.

\section{Ethical considerations}

The ethical approval for the study was obtained from the Institutional Ethics and Scientific Review Committee, Vice Deanship for Postgraduate Studies and Scientific Research, College of Dentistry, Imam Abdulrahman Bin Faisal University, Dammam, Kingdom of Saudi Arabia, vide approval number EA:202048, dated: 26/03/2020. Participation in the study was voluntary and respondents were maintained anonymity.

\section{Statistical analysis}

Obtained data were coded, tabulated and analyzed using Statistical Package for Social Sciences (IBM SPSS Version 20). The age of the participants was grouped as those below 35 years of age and those aged 35 years and above. Imputation was done for questions having missing responses. Participants who had provided complete demographic data but had few missing data for the study variables were included for imputation. This was done using the linear interpolation method, which uses values from adjacent data points to impute the missing values. The imputed values were rounded off to the nearest integer. Participants with missing demographic data were completely excluded from the analysis. Descriptive statistics were expressed as frequency and percentages. Bivariate analysis was done using the Chi-square test with the country of work and age group as independent variables. A $p$-value of $<0.05$ was considered statistically significant.

\section{Results}

The response rate was $77.1 \%$ with 1022 respondents. Incomplete entries were rejected and a total of 788 complete responses were included for the study. About $60 \%$ of the respondents were aged less than 35 years and over half of them were females (55.5\%). The respondents were from India (47\%), Malaysia (9.4\%), Gulf Cooperation Council (GCC) (39.1\%) and other Asian countries (4.6\%). Almost 70\% of the study participants were from the dental fraternity $(n=566)$ - and the other $30 \%$ belonged to general HCPs (Table 1 and 2).

\section{Job-related concerns during the pandemic}

The first domain identified the concerns regarding their work during the pandemic (Table 3 ). More than half of the respondents (63\%) in every country felt that they were not afraid of telling their family regarding the potential risks exposed during their work. Less than a quarter of them felt otherwise. 
Table 1. Characteristics of study population

\begin{tabular}{|c|c|c|}
\hline Variable & Characteristics & n $(\%)$ \\
\hline \multirow{2}{*}{ Age group } & Less than 35 years & $469(59.5 \%)$ \\
\hline & 35 years or more & $319(40.5 \%)$ \\
\hline \multirow{2}{*}{ Gender } & Male & $351(44.5 \%)$ \\
\hline & Female & $437(55.5 \%)$ \\
\hline \multirow{4}{*}{ Country of work } & India & $370(47 \%)$ \\
\hline & Malaysia & $74(9.4 \%)$ \\
\hline & $\mathrm{GCC}$ & $308(39.1 \%)$ \\
\hline & Others* & $36(4.6 \%)$ \\
\hline \multirow{2}{*}{ Field of work } & Medical Professionals & $222(28.2 \%)$ \\
\hline & Dental Professionals & $566(71.8 \%)$ \\
\hline
\end{tabular}

*Singapore, Indonesia, United States of America, Egypt (Health care professionals on official deputations)

Table 2. Responses of study participants

\begin{tabular}{|c|c|c|c|c|c|c|}
\hline Domains and questions & $\mathrm{n}$ & $\%$ & $\mathrm{n}$ & $\%$ & $\mathrm{n}$ & $\%$ \\
\hline Work / job related concerns & \multicolumn{2}{|c|}{ Yes } & \multicolumn{2}{|c|}{ Somewhat } & \multicolumn{2}{|c|}{ No } \\
\hline $\begin{array}{l}\text { Do you feel afraid of telling your family about the potential risk you are exposed at your } \\
\text { clinic? }\end{array}$ & 125 & 15.9 & 165 & 20.9 & 498 & 63.2 \\
\hline Do you have the perception that people would avoid you because of your job? & 173 & 22.0 & 262 & 33.2 & 353 & 44.8 \\
\hline Do you feel people will distance away your family members because of your job? & 129 & 16.4 & 202 & 25.6 & 457 & 58.0 \\
\hline $\begin{array}{l}\text { Do have concerns about inadequate staff at your workplace to handle the increased } \\
\text { demand? }\end{array}$ & 338 & 42.9 & 177 & 22.5 & 273 & 34.6 \\
\hline Do you have concerns that there would be more conflict amongst colleagues at work? & 245 & 31.1 & 231 & 29.3 & 312 & 39.6 \\
\hline Do you feel you would have an increase in workload? & 295 & 37.4 & 176 & 22.3 & 317 & 40.2 \\
\hline Have you changed your behaviour fearing being infected at workplace? & 361 & 45.8 & 214 & 27.2 & 213 & 27.0 \\
\hline Do you appreciate job related risk compensation from your stakeholders' post pandemic? & 428 & 54.3 & 191 & 24.2 & 169 & 21.4 \\
\hline Have you felt that you had to do your job as it was your professional and ethical duty? & 682 & 86.5 & 71 & 9.0 & 35 & 4.4 \\
\hline $\begin{array}{l}\text { Do you appreciate your self-recognition in dealing with a pandemic like COVID-19 and } \\
\text { confident to educate your peer? }\end{array}$ & 637 & 80.8 & 128 & 16.2 & 23 & 2.9 \\
\hline Perception of stress & \multicolumn{2}{|c|}{ Yes } & \multicolumn{2}{|c|}{ Somewhat } & \multicolumn{2}{|c|}{ No } \\
\hline Do you feel more stressed at work? & 287 & 36.4 & 254 & 32.2 & 247 & 31.3 \\
\hline Are you stressed seeing your colleagues getting infected/treated due to COVID $-19 ?$ & 419 & 53.2 & 225 & 28.6 & 144 & 18.3 \\
\hline Are you stressed assuming that you could transmit COVID -19 to your family or friends? & 509 & 64.6 & 194 & 24.6 & 85 & 10.8 \\
\hline Are you stressed not knowing when the COVID -19 outbreak will be under control? & 534 & 67.8 & 189 & 24.0 & 65 & 8.2 \\
\hline Are you stressed seeing colleagues displaying COVID -19 like similar symptoms? & 376 & 47.7 & 255 & 32.4 & 157 & 19.9 \\
\hline Are you stressed seeing your colleagues stressed or afraid of COVID $-19 ?$ & 399 & 50.6 & 258 & 32.7 & 131 & 16.6 \\
\hline Preparedness & \multicolumn{2}{|c|}{ Yes } & \multicolumn{2}{|c|}{$\begin{array}{c}\text { Inadequate / } \\
\text { fewer }\end{array}$} & \multicolumn{2}{|c|}{ No } \\
\hline Have you received specialized training for containing the contagious diseases? & 203 & 25.8 & 289 & 36.7 & 296 & 37.6 \\
\hline $\begin{array}{l}\text { Are you and your colleagues and staff well trained in infection control at your clinic or } \\
\text { workplace? }\end{array}$ & 408 & 51.8 & 271 & 34.4 & 109 & 13.8 \\
\hline $\begin{array}{l}\text { Does your clinic have adequate infection control or personal protective equipment and } \\
\text { material? }\end{array}$ & 403 & 51.1 & 264 & 33.5 & 121 & 15.4 \\
\hline $\begin{array}{l}\text { Do you have clear guidelines from Hospital/ health department for infection prevention at } \\
\text { work? }\end{array}$ & 529 & 67.1 & 174 & 22.1 & 85 & 10.8 \\
\hline
\end{tabular}


Table 3. Country-wise, age group wise and profession wise response to job related concerns during pandemic

\begin{tabular}{|c|c|c|c|c|c|c|c|c|c|c|c|c|}
\hline \multirow[b]{2}{*}{ Question } & \multirow[b]{2}{*}{ Response } & \multicolumn{5}{|c|}{ Country of work $n(\%)$} & \multicolumn{3}{|c|}{ Age group n (\%) } & \multicolumn{3}{|c|}{ Profession n (\%) } \\
\hline & & India & Malaysia & $\mathrm{GCC}$ & Others & $\mathrm{p}$ value & $\begin{array}{c}\text { Less than } \\
35 \text { years }\end{array}$ & $\begin{array}{c}35 \text { years } \\
\text { and above }\end{array}$ & $\mathrm{p}$ value & Medical & Dental & $p$ value \\
\hline \multirow{3}{*}{$\begin{array}{l}\text { Do you feel afraid of telling } \\
\text { your family about the potential } \\
\text { risk you are exposed at your } \\
\text { clinic? }\end{array}$} & Yes & $\begin{array}{c}46 \\
(12.4 \%)\end{array}$ & $\begin{array}{c}10 \\
(13.5 \%)\end{array}$ & $\begin{array}{c}62 \\
(20.1 \%)\end{array}$ & $\begin{array}{c}7 \\
(19.4 \%)\end{array}$ & \multirow{3}{*}{0.005} & $\begin{array}{c}77 \\
(16.4 \%)\end{array}$ & $\begin{array}{c}48 \\
(15.0 \%)\end{array}$ & & $\begin{array}{c}50 \\
(22.5 \%)\end{array}$ & $\begin{array}{c}75 \\
(13.3 \%)\end{array}$ & \multirow{3}{*}{0.004} \\
\hline & Somewhat & $\begin{array}{c}67 \\
(18.1 \%)\end{array}$ & $\begin{array}{c}12 \\
(16.2 \%)\end{array}$ & $\begin{array}{c}76 \\
(24.7 \%)\end{array}$ & $\begin{array}{c}10 \\
(27.8 \%)\end{array}$ & & $\begin{array}{c}107 \\
(22.8 \%)\end{array}$ & $\begin{array}{c}58 \\
(18.2 \%)\end{array}$ & 0.197 & $\begin{array}{c}47 \\
(21.2 \%)\end{array}$ & $\begin{array}{c}118 \\
(20.8 \%)\end{array}$ & \\
\hline & No & $\begin{array}{c}257 \\
(69.5 \%) \\
\end{array}$ & $\begin{array}{c}52 \\
(70.3 \%) \\
\end{array}$ & $\begin{array}{c}170 \\
(55.2 \%) \\
\end{array}$ & $\begin{array}{c}19 \\
(52.8 \%) \\
\end{array}$ & & $\begin{array}{c}285 \\
(60.8 \%) \\
\end{array}$ & $\begin{array}{c}213 \\
(66.8 \%) \\
\end{array}$ & & $\begin{array}{c}125 \\
(56.3 \%) \\
\end{array}$ & $\begin{array}{c}373 \\
(65.99 \%) \\
\end{array}$ & \\
\hline \multirow{3}{*}{$\begin{array}{l}\text { Do you have the perception that } \\
\text { people would avoid you because } \\
\text { of your job? }\end{array}$} & Yes & $\begin{array}{c}59 \\
(15.9 \%)\end{array}$ & $\begin{array}{c}12 \\
(16.2 \%)\end{array}$ & $\begin{array}{c}92 \\
(29.9 \%)\end{array}$ & $\begin{array}{c}10 \\
(27.8 \%)\end{array}$ & \multirow{3}{*}{$<0.001$} & $\begin{array}{c}107 \\
(22.8 \%)\end{array}$ & $\begin{array}{c}66 \\
(20.7 \%)\end{array}$ & & $\begin{array}{c}73 \\
(32.9 \%)\end{array}$ & $\begin{array}{c}100 \\
(17.7 \%)\end{array}$ & \multirow{3}{*}{$<0.001$} \\
\hline & Somewhat & $\begin{array}{c}119 \\
(32.2 \%)\end{array}$ & $\begin{array}{c}23 \\
(31.1 \%)\end{array}$ & $\begin{array}{c}107 \\
(34.7 \%)\end{array}$ & $\begin{array}{c}13 \\
(36.1 \%)\end{array}$ & & $\begin{array}{c}160 \\
(34.1 \%)\end{array}$ & $\begin{array}{c}102 \\
(32.0 \%)\end{array}$ & 0.491 & $\begin{array}{c}66 \\
(29.7 \%)\end{array}$ & $\begin{array}{c}196 \\
(34.6 \%)\end{array}$ & \\
\hline & No & $\begin{array}{c}192 \\
(51.9 \%) \\
\end{array}$ & $\begin{array}{c}39 \\
(52.7 \%) \\
\end{array}$ & $\begin{array}{c}109 \\
(35.4 \%) \\
\end{array}$ & $\begin{array}{c}13 \\
(36.1 \%) \\
\end{array}$ & & $\begin{array}{c}202 \\
(43.1 \%)\end{array}$ & $\begin{array}{c}151 \\
(47.3 \%) \\
\end{array}$ & & $\begin{array}{c}83 \\
(37.4 \%) \\
\end{array}$ & $\begin{array}{c}270 \\
(47.7 \%) \\
\end{array}$ & \\
\hline \multirow{3}{*}{$\begin{array}{l}\text { Do you feel people will distance } \\
\text { away your family members } \\
\text { because of your job? }\end{array}$} & Yes & $\begin{array}{c}55 \\
(14.9 \%)\end{array}$ & $\begin{array}{c}12 \\
(16.2 \%)\end{array}$ & $\begin{array}{c}56 \\
(18.2 \%)\end{array}$ & $\begin{array}{c}6 \\
(16.7 \%)\end{array}$ & \multirow{3}{*}{0.489} & $\begin{array}{c}76 \\
(16.2 \%)\end{array}$ & $\begin{array}{c}53 \\
(16.6 \%)\end{array}$ & & $\begin{array}{c}54 \\
(24.3 \%)\end{array}$ & $\begin{array}{c}75 \\
(13.3 \%)\end{array}$ & \multirow{3}{*}{0.001} \\
\hline & Somewhat & $\begin{array}{c}102 \\
(27.6 \%)\end{array}$ & $\begin{array}{c}13 \\
(17.6 \%)\end{array}$ & $\begin{array}{c}80 \\
(26.0 \%)\end{array}$ & $\begin{array}{c}7 \\
(19.4 \%)\end{array}$ & & $\begin{array}{c}118 \\
(25.2 \%)\end{array}$ & $\begin{array}{c}84 \\
(26.3 \%)\end{array}$ & 0.903 & $\begin{array}{c}53 \\
(23.9 \%)\end{array}$ & $\begin{array}{c}149 \\
(26.3 \%)\end{array}$ & \\
\hline & No & $\begin{array}{c}213 \\
(57.6 \%) \\
\end{array}$ & $\begin{array}{c}49 \\
(66.2 \%) \\
\end{array}$ & $\begin{array}{c}172 \\
(55.8 \%) \\
\end{array}$ & $\begin{array}{c}23 \\
(63.9 \%) \\
\end{array}$ & & $\begin{array}{c}275 \\
(58.6 \%) \\
\end{array}$ & $\begin{array}{c}182 \\
(57.1 \%) \\
\end{array}$ & & $\begin{array}{c}115 \\
(51.8 \%) \\
\end{array}$ & $\begin{array}{c}342 \\
(60.4 \%) \\
\end{array}$ & \\
\hline \multirow{3}{*}{$\begin{array}{l}\text { Do have concerns about } \\
\text { inadequate staff at your } \\
\text { workplace to handle the } \\
\text { increased demand? }\end{array}$} & Yes & $\begin{array}{c}165 \\
(44.6 \%)\end{array}$ & $\begin{array}{c}37 \\
(50.0 \%)\end{array}$ & $\begin{array}{c}119 \\
(38.6 \%)\end{array}$ & $\begin{array}{c}17 \\
(47.2 \%)\end{array}$ & \multirow{3}{*}{0.380} & $\begin{array}{c}195 \\
(41.6 \%)\end{array}$ & $\begin{array}{c}143 \\
(44.8 \%)\end{array}$ & & $\begin{array}{c}118 \\
(53.2 \%)\end{array}$ & $\begin{array}{c}220 \\
(38.9 \%)\end{array}$ & \multirow{3}{*}{$<0.001$} \\
\hline & Somewhat & $\begin{array}{c}74 \\
(20.0 \%)\end{array}$ & $\begin{array}{c}15 \\
(20.3 \%)\end{array}$ & $\begin{array}{c}79 \\
(25.6 \%)\end{array}$ & $\begin{array}{c}9 \\
(25.0 \%)\end{array}$ & & $\begin{array}{c}114 \\
(24.3 \%)\end{array}$ & $\begin{array}{c}63 \\
(19.7 \%)\end{array}$ & 0.314 & $\begin{array}{c}53 \\
(23.9 \%)\end{array}$ & $\begin{array}{c}124 \\
(21.9 \%)\end{array}$ & \\
\hline & No & $\begin{array}{c}131 \\
(35.4 \%)\end{array}$ & $\begin{array}{c}22 \\
(29.7 \%)\end{array}$ & $\begin{array}{c}110 \\
(35.7 \%)\end{array}$ & $\begin{array}{c}10 \\
(27.8 \%)\end{array}$ & & $\begin{array}{c}160 \\
(34.1 \%)\end{array}$ & $\begin{array}{c}113 \\
(35.4 \%)\end{array}$ & & $\begin{array}{c}51 \\
(23 \%)\end{array}$ & $\begin{array}{c}222 \\
(39.2 \%)\end{array}$ & \\
\hline \multirow{3}{*}{$\begin{array}{l}\text { Do you have concerns that there } \\
\text { would be more conflict amongst } \\
\text { colleagues at work? }\end{array}$} & Yes & $\begin{array}{c}106 \\
(28.6 \%)\end{array}$ & $\begin{array}{c}22 \\
(29.7 \%)\end{array}$ & $\begin{array}{c}110 \\
(35.7 \%)\end{array}$ & $\begin{array}{c}7 \\
(19.4 \%)\end{array}$ & \multirow{3}{*}{0.088} & $\begin{array}{c}141 \\
(30.1 \%)\end{array}$ & $\begin{array}{c}104 \\
(32.6 \%)\end{array}$ & & $\begin{array}{c}83 \\
(37.4 \%)\end{array}$ & $\begin{array}{c}162 \\
(28.6 \%)\end{array}$ & \multirow{3}{*}{0.053} \\
\hline & Somewhat & $\begin{array}{c}117 \\
(31.6 \%)\end{array}$ & $\begin{array}{c}16 \\
(21.6 \%)\end{array}$ & $\begin{array}{c}83 \\
(26.9 \%)\end{array}$ & $\begin{array}{c}15 \\
(41.7 \%)\end{array}$ & & $\begin{array}{c}139 \\
(29.6 \%)\end{array}$ & $\begin{array}{c}92 \\
(28.8 \%)\end{array}$ & 0.748 & $\begin{array}{c}57 \\
(25.7 \%)\end{array}$ & $\begin{array}{c}174 \\
(30.7 \%)\end{array}$ & \\
\hline & No & $\begin{array}{c}147 \\
(39.7 \%)\end{array}$ & $\begin{array}{c}36 \\
(48.6 \%)\end{array}$ & $\begin{array}{c}115 \\
(37.3 \%)\end{array}$ & $\begin{array}{c}14 \\
(38.9 \%)\end{array}$ & & $\begin{array}{c}189 \\
(40.3 \%)\end{array}$ & $\begin{array}{c}123 \\
(38.6 \%)\end{array}$ & & $\begin{array}{c}82 \\
(36.9 \%)\end{array}$ & $\begin{array}{c}230 \\
(40.6 \%)\end{array}$ & \\
\hline \multirow{4}{*}{$\begin{array}{l}\text { Do you feel you would have an } \\
\text { increase in workload? }\end{array}$} & Yes & $\begin{array}{c}134 \\
(36.2 \%)\end{array}$ & $\begin{array}{c}31 \\
(41.9 \%)\end{array}$ & $\begin{array}{c}116 \\
(37.7 \%)\end{array}$ & $\begin{array}{c}14 \\
(38.9 \%)\end{array}$ & \multirow{3}{*}{0.494} & $\begin{array}{c}182 \\
(38.8 \%)\end{array}$ & $\begin{array}{c}113 \\
(35.4 \%)\end{array}$ & & $\begin{array}{c}114 \\
(51.4 \%)\end{array}$ & $\begin{array}{c}181 \\
(32.0 \%)\end{array}$ & \multirow{3}{*}{$<0.001$} \\
\hline & Somewhat & $\begin{array}{c}76 \\
(20.5 \%)\end{array}$ & $\begin{array}{c}19 \\
(25.7 \%)\end{array}$ & $\begin{array}{c}70 \\
(22.7 \%)\end{array}$ & $\begin{array}{c}11 \\
(30.6 \%)\end{array}$ & & $\begin{array}{c}104 \\
(22.2 \%)\end{array}$ & $\begin{array}{c}72 \\
(22.6 \%)\end{array}$ & 0.602 & $\begin{array}{c}52 \\
(23.4 \%)\end{array}$ & $\begin{array}{c}124 \\
(21.9 \%)\end{array}$ & \\
\hline & No & $\begin{array}{c}160 \\
(43.2 \%)\end{array}$ & $\begin{array}{c}24 \\
(32.4 \%)\end{array}$ & $\begin{array}{c}122 \\
(39.6 \%)\end{array}$ & $\begin{array}{c}11 \\
(30.6 \%)\end{array}$ & & $\begin{array}{c}183 \\
(39.0 \%)\end{array}$ & $\begin{array}{c}134 \\
(42.0 \%)\end{array}$ & & $\begin{array}{c}56 \\
(25.2 \%)\end{array}$ & $\begin{array}{c}261 \\
(46.1 \%)\end{array}$ & \\
\hline & Yes & $\begin{array}{c}140 \\
(37.8 \%)\end{array}$ & $\begin{array}{c}43 \\
(58.1 \%)\end{array}$ & $\begin{array}{c}161 \\
(52.3 \%)\end{array}$ & $\begin{array}{c}17 \\
(47.2 \%)\end{array}$ & 0.001 & $\begin{array}{c}209 \\
(44.6 \%)\end{array}$ & $\begin{array}{c}152 \\
(47.6 \%)\end{array}$ & 0.220 & $\begin{array}{c}113 \\
(50.9 \%)\end{array}$ & $\begin{array}{c}248 \\
(43.8 \%)\end{array}$ & 0.039 \\
\hline
\end{tabular}




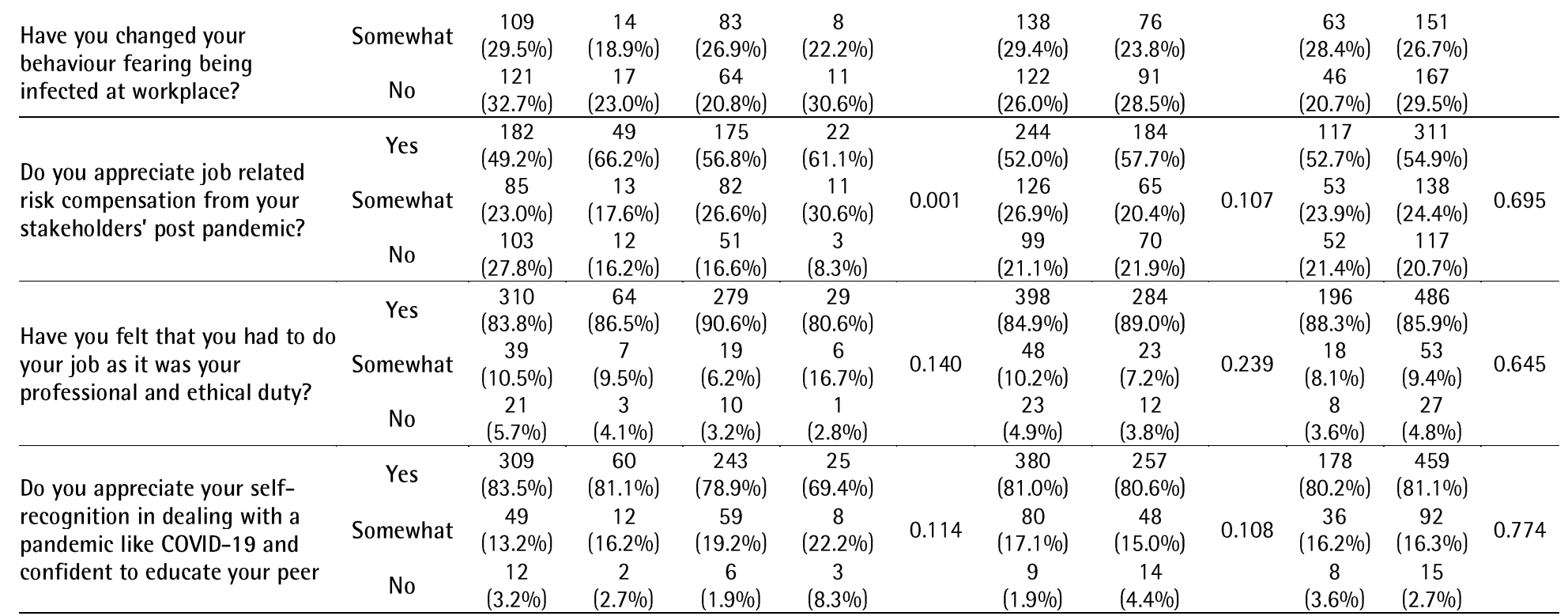

\section{Differences based on geographical location and profession}

Almost 50\% of the participants from India and Malaysia perceived that they would face stigma from the society because of their job $(p<0.001)$. Across countries, it was found that the participants felt inadequate workforce to handle the increased demand $(p=0.380)$. Change of behavior fearing infection was highest among respondents from Malaysia (58.1\%) and lowest among respondents from India (37.8\%). The majority of the respondents across countries ( $>80 \%)$ felt that it was their ethical duty to work during this pandemic $(p=0.140)$. There were no significant differences observed between age groups in the domain of job-related concerns during the pandemic $(p>0.1)$. Analyzing the response in relation to profession, there was a significant job-related concern among the medical professionals compared to dental professionals (Most of the $p$ values were $<0.05$-Table 3 ).

Perception of stress during the pandemic

Stress was a common phenomenon observed in all the participants studied (Table 4). The stress factor depended greatly on the cultural set up, age and professional setting.

Table 4. Country-wise, age group wise and profession wise response to perception of stress during pandemic

\begin{tabular}{|c|c|c|c|c|c|c|c|c|c|c|c|c|}
\hline \multirow[b]{2}{*}{ Question } & \multirow[b]{2}{*}{ Response } & \multicolumn{5}{|c|}{ Country of work $n(\%)$} & \multicolumn{3}{|c|}{ Age group n (\%) } & \multicolumn{3}{|c|}{ Profession n (\%) } \\
\hline & & India & Malaysia & $\mathrm{GCC}$ & Others & $\mathrm{p}$ value & $\begin{array}{l}\text { Less than } \\
35 \text { years }\end{array}$ & $\begin{array}{l}35 \text { years } \\
\text { and above }\end{array}$ & $p$ value & Medical & Dental & $p$ value \\
\hline \multirow{3}{*}{$\begin{array}{l}\text { Do you feel more } \\
\text { stressed at work? }\end{array}$} & Yes & $\begin{array}{c}111 \\
(30.0 \%)\end{array}$ & $\begin{array}{c}13 \\
(17.6 \%)\end{array}$ & $\begin{array}{c}149 \\
(48.4 \%)\end{array}$ & $\begin{array}{c}14 \\
(38.9 \%)\end{array}$ & \multirow{3}{*}{$<0.001$} & $\begin{array}{c}147 \\
(31.3 \%)\end{array}$ & $\begin{array}{c}140 \\
(43.9 \%)\end{array}$ & \multirow{3}{*}{0.002} & $\begin{array}{c}95 \\
(42.8 \%)\end{array}$ & $\begin{array}{c}192 \\
(33.9 \%)\end{array}$ & \multirow{3}{*}{0.066} \\
\hline & Somewhat & $\begin{array}{c}119 \\
(32.2 \%)\end{array}$ & $\begin{array}{c}32 \\
(43.2 \%)\end{array}$ & $\begin{array}{c}89 \\
(28.9 \%)\end{array}$ & $\begin{array}{c}14 \\
(38.9 \%)\end{array}$ & & $\begin{array}{c}164 \\
(35.0 \%)\end{array}$ & $\begin{array}{c}90 \\
(28.2 \%)\end{array}$ & & $\begin{array}{c}65 \\
(29.3 \%)\end{array}$ & $\begin{array}{c}189 \\
(33.4 \%)\end{array}$ & \\
\hline & No & $\begin{array}{c}140 \\
(37.8 \%)\end{array}$ & $\begin{array}{c}29 \\
(39.2 \%)\end{array}$ & $\begin{array}{c}70 \\
(22.7 \%)\end{array}$ & $\begin{array}{c}8 \\
(22.2 \%)\end{array}$ & & $\begin{array}{c}158 \\
(33.7 \%)\end{array}$ & $\begin{array}{c}89 \\
(27.9 \%)\end{array}$ & & $\begin{array}{c}62 \\
(27.9 \%)\end{array}$ & $\begin{array}{c}185 \\
(32.7 \%)\end{array}$ & \\
\hline \multirow{3}{*}{$\begin{array}{l}\text { Are you stressed } \\
\text { seeing your } \\
\text { colleagues getting } \\
\text { infected/treated } \\
\text { due to COVID -19? }\end{array}$} & Yes & $\begin{array}{c}187 \\
(50.5 \%)\end{array}$ & $\begin{array}{c}38 \\
(51.4 \%)\end{array}$ & $\begin{array}{c}171 \\
(55.5 \%)\end{array}$ & $\begin{array}{c}23 \\
(63.9 \%)\end{array}$ & \multirow{3}{*}{0.556} & $\begin{array}{c}237 \\
(50.5 \%)\end{array}$ & $\begin{array}{c}182 \\
(57.1 \%)\end{array}$ & \multirow{3}{*}{0.147} & $\begin{array}{c}137 \\
(61.7 \%)\end{array}$ & $\begin{array}{c}282 \\
(49.8 \%)\end{array}$ & \multirow{3}{*}{0.009} \\
\hline & Somewhat & $\begin{array}{c}107 \\
(28.9 \%)\end{array}$ & $\begin{array}{c}23 \\
(31.1 \%)\end{array}$ & $\begin{array}{c}88 \\
(28.6 \%)\end{array}$ & $\begin{array}{c}7 \\
(19.4 \%)\end{array}$ & & $\begin{array}{c}145 \\
(30.9 \%)\end{array}$ & $\begin{array}{c}80 \\
(25.1 \%)\end{array}$ & & $\begin{array}{c}54 \\
(24.3 \%)\end{array}$ & $\begin{array}{c}171 \\
(30.2 \%)\end{array}$ & \\
\hline & No & $\begin{array}{c}76 \\
(20.5 \%)\end{array}$ & $\begin{array}{c}13 \\
(17.6 \%)\end{array}$ & $\begin{array}{c}49 \\
(15.9 \%)\end{array}$ & $\begin{array}{c}6 \\
(16.7 \%)\end{array}$ & & $\begin{array}{c}87 \\
(18.6 \%)\end{array}$ & $\begin{array}{c}57 \\
(17.9 \%)\end{array}$ & & $\begin{array}{c}31 \\
(14 \%)\end{array}$ & $\begin{array}{c}113 \\
(20 \%)\end{array}$ & \\
\hline \multirow{2}{*}{$\begin{array}{l}\text { Are you stressed } \\
\text { assuming that you } \\
\text { could transmit } \\
\text { COVID }-19 \text { to your } \\
\text { family or friends? }\end{array}$} & Yes & $\begin{array}{c}205 \\
(55.4 \%)\end{array}$ & $\begin{array}{c}49 \\
(66.2 \%)\end{array}$ & $\begin{array}{c}231 \\
(75.0 \%)\end{array}$ & $\begin{array}{c}24 \\
(66.7 \%)\end{array}$ & \multirow[b]{2}{*}{$<0.001$} & $\begin{array}{c}302 \\
(64.4 \%)\end{array}$ & $\begin{array}{c}207 \\
(64.9 \%)\end{array}$ & \multirow[b]{2}{*}{0.584} & $\begin{array}{c}160 \\
(72.1 \%)\end{array}$ & $\begin{array}{c}349 \\
(61.7 \%)\end{array}$ & \multirow[b]{2}{*}{0.019} \\
\hline & Somewhat & $\begin{array}{c}115 \\
(31.1 \%)\end{array}$ & $\begin{array}{c}14 \\
(18.9 \%)\end{array}$ & $\begin{array}{c}54 \\
(17.5 \%)\end{array}$ & $\begin{array}{c}11 \\
(30.6 \%)\end{array}$ & & $\begin{array}{c}120 \\
(25.6 \%)\end{array}$ & $\begin{array}{c}74 \\
(23.2 \%)\end{array}$ & & $\begin{array}{c}41 \\
(18.5 \%)\end{array}$ & $\begin{array}{c}153 \\
(27 \%)\end{array}$ & \\
\hline
\end{tabular}




\begin{tabular}{|c|c|c|c|c|c|c|c|c|c|c|c|c|}
\hline & No & $\begin{array}{c}50 \\
(13.5 \%)\end{array}$ & $\begin{array}{c}11 \\
(14.9 \%)\end{array}$ & $\begin{array}{c}23 \\
(7.5 \%)\end{array}$ & $\begin{array}{c}1 \\
(2.8 \%)\end{array}$ & & $\begin{array}{c}47 \\
(10.0 \%)\end{array}$ & $\begin{array}{c}38 \\
(11.9 \%)\end{array}$ & & $\begin{array}{c}21 \\
(9.5 \%)\end{array}$ & $\begin{array}{c}64 \\
(11.3 \%)\end{array}$ & \\
\hline \multirow{3}{*}{$\begin{array}{l}\text { Are you stressed } \\
\text { not knowing when } \\
\text { the COVID - } 19 \\
\text { outbreak will be } \\
\text { under control? }\end{array}$} & Yes & $\begin{array}{c}249 \\
(67.3 \%)\end{array}$ & $\begin{array}{c}51 \\
(68.9 \%)\end{array}$ & $\begin{array}{c}212 \\
(68.8 \%)\end{array}$ & $\begin{array}{c}22 \\
(61.1 \%)\end{array}$ & \multirow{3}{*}{0.128} & $\begin{array}{c}313 \\
(66.7 \%)\end{array}$ & $\begin{array}{c}221 \\
(69.3 \%)\end{array}$ & & $\begin{array}{c}155 \\
(69.8 \%)\end{array}$ & $\begin{array}{c}379 \\
(67 \%)\end{array}$ & \multirow{3}{*}{0.741} \\
\hline & Somewhat & $\begin{array}{c}90 \\
(24.3 \%)\end{array}$ & $\begin{array}{c}12 \\
(16.2 \%)\end{array}$ & $\begin{array}{c}78 \\
(25.3 \%)\end{array}$ & $\begin{array}{c}9 \\
(25.0 \%)\end{array}$ & & $\begin{array}{c}117 \\
(24.9 \%)\end{array}$ & $\begin{array}{c}72 \\
(22.6 \%)\end{array}$ & 0.728 & $\begin{array}{c}50 \\
(22.5 \%)\end{array}$ & $\begin{array}{c}139 \\
(24.6 \%)\end{array}$ & \\
\hline & No & $\begin{array}{c}31 \\
(8.4 \%)\end{array}$ & $\begin{array}{c}11 \\
(14.9 \%)\end{array}$ & $\begin{array}{c}18 \\
(5.8 \%)\end{array}$ & $\begin{array}{c}5 \\
(13.9 \%)\end{array}$ & & $\begin{array}{c}39 \\
(8.3 \%)\end{array}$ & $\begin{array}{c}26 \\
(8.2 \%)\end{array}$ & & $\begin{array}{c}17 \\
(7.7 \%)\end{array}$ & $\begin{array}{c}48 \\
(8.5 \%)\end{array}$ & \\
\hline \multirow{3}{*}{$\begin{array}{l}\text { Are you stressed } \\
\text { seeing colleagues } \\
\text { displaying COVID - } \\
19 \text { like similar } \\
\text { symptoms? }\end{array}$} & Yes & $\begin{array}{c}157 \\
(42.4 \%)\end{array}$ & $\begin{array}{c}40 \\
(54.1 \%)\end{array}$ & $\begin{array}{c}160 \\
(51.9 \%)\end{array}$ & $\begin{array}{c}19 \\
(52.8 \%)\end{array}$ & \multirow{3}{*}{0.004} & $\begin{array}{c}216 \\
(46.1 \%)\end{array}$ & $\begin{array}{c}160 \\
(50.2 \%)\end{array}$ & & $\begin{array}{c}125 \\
(56.3 \%)\end{array}$ & $\begin{array}{c}251 \\
(44.3 \%)\end{array}$ & \multirow{3}{*}{0.006} \\
\hline & Somewhat & $\begin{array}{c}116 \\
(31.4 \%)\end{array}$ & $\begin{array}{c}21 \\
(28.4 \%)\end{array}$ & $\begin{array}{c}106 \\
(34.4 \%)\end{array}$ & $\begin{array}{c}12 \\
(33.3 \%)\end{array}$ & & $\begin{array}{c}159 \\
(33.9 \%)\end{array}$ & $\begin{array}{c}96 \\
(30.1 \%)\end{array}$ & 0.466 & $\begin{array}{c}65 \\
(29.3 \%)\end{array}$ & $\begin{array}{c}190 \\
(33.6 \%)\end{array}$ & \\
\hline & No & $\begin{array}{c}97 \\
(26.2 \%)\end{array}$ & $\begin{array}{c}13 \\
(17.6 \%)\end{array}$ & $\begin{array}{c}42 \\
(13.6 \%)\end{array}$ & $\begin{array}{c}5 \\
(13.9 \%)\end{array}$ & & $\begin{array}{c}94 \\
(20.0 \%)\end{array}$ & $\begin{array}{c}63 \\
(19.7 \%)\end{array}$ & & $\begin{array}{c}32 \\
(14.4 \%)\end{array}$ & $\begin{array}{c}125 \\
(22.1 \%)\end{array}$ & \\
\hline \multirow{3}{*}{$\begin{array}{l}\text { Are you stressed } \\
\text { seeing your } \\
\text { colleagues stressed } \\
\text { or afraid of COVID } \\
-19 ?\end{array}$} & Yes & $\begin{array}{c}176 \\
(47.6 \%)\end{array}$ & $\begin{array}{c}37 \\
(50.0 \%)\end{array}$ & $\begin{array}{c}166 \\
(53.9 \%)\end{array}$ & $\begin{array}{c}20 \\
(55.6 \%)\end{array}$ & & $\begin{array}{c}232 \\
(49.5 \%)\end{array}$ & $\begin{array}{c}167 \\
(52.4 \%)\end{array}$ & & $\begin{array}{c}129 \\
(58.1 \%)\end{array}$ & $\begin{array}{c}270 \\
(47.7 \%)\end{array}$ & \multirow{3}{*}{0.023} \\
\hline & Somewhat & $\begin{array}{c}129 \\
(34.9 \%)\end{array}$ & $\begin{array}{c}22 \\
(29.7 \%)\end{array}$ & $\begin{array}{c}97 \\
(31.5 \%)\end{array}$ & $\begin{array}{c}10 \\
(27.8 \%)\end{array}$ & 0.652 & $\begin{array}{c}153 \\
(32.6 \%)\end{array}$ & $\begin{array}{c}105 \\
(32.9 \%)\end{array}$ & 0.480 & $\begin{array}{c}65 \\
(29.3 \%)\end{array}$ & $\begin{array}{c}193 \\
(34.1 \%)\end{array}$ & \\
\hline & No & $\begin{array}{c}65 \\
(17.6 \%)\end{array}$ & $\begin{array}{c}15 \\
(20.3 \%)\end{array}$ & $\begin{array}{c}45 \\
(14.6 \%)\end{array}$ & $\begin{array}{c}6 \\
(16.7 \%)\end{array}$ & & $\begin{array}{c}84 \\
(17.9 \%)\end{array}$ & $\begin{array}{c}47 \\
(14.77 \%)\end{array}$ & & $\begin{array}{c}28 \\
(12.6 \%)\end{array}$ & $\begin{array}{c}103 \\
(18.2 \%)\end{array}$ & \\
\hline
\end{tabular}

\section{Differences based on geographical location}

It was observed that respondents from Gulf countries felt more stressed at work (48.4\%), compared to other countries, especially Malaysia (17.6\%). About three-quarters of the respondents from Gulf Cooperation Council countries felt that they were stressed with the thought of transmitting COVID-19 to family or friends if infected $(p<0.001)$.

\section{Differences based on profession and age}

The perceived stress at the workplace was high (43.9\%) for people aged 35 years and above. ( $p=$ 0.002). The medical professionals were more stressed at seeing their colleagues getting infected (61.7\%), assuming that they could transmit COVID 19 to their family or friends $(72.1 \%)$ and seeing colleagues displaying symptoms (56.3\%); compared to the dental professionals.

\section{Preparedness during pandemic (table 5)}

Table 5. Country-wise, age group wise and profession wise response to preparedness during pandemic

\begin{tabular}{|c|c|c|c|c|c|c|c|c|c|c|c|c|}
\hline \multirow[b]{2}{*}{ Question } & \multirow[b]{2}{*}{ Response } & \multicolumn{5}{|c|}{ Country of work $n(\%)$} & \multicolumn{3}{|c|}{ Age group n (\%) } & \multicolumn{3}{|c|}{ Profession n (\%) } \\
\hline & & India & Malaysia & GCC & Others & $p$ value & $\begin{array}{r}\text { Less than } \\
35 \text { years }\end{array}$ & $\begin{array}{l}35 \text { years } \\
\text { and above }\end{array}$ & $\mathrm{p}$ value & Medical & Dental & $p$ value \\
\hline \multirow{3}{*}{$\begin{array}{l}\text { Have you received } \\
\text { specialized training } \\
\text { for containing the } \\
\text { contagious diseases? }\end{array}$} & Yes & $\begin{array}{c}68 \\
(18.4 \%)\end{array}$ & $\begin{array}{c}20 \\
(27.0 \%)\end{array}$ & $\begin{array}{c}104 \\
(33.8 \%)\end{array}$ & $\begin{array}{c}11 \\
(30.6 \%)\end{array}$ & & $\begin{array}{c}120 \\
(25.6 \%)\end{array}$ & $\begin{array}{c}83 \\
(26.0 \%)\end{array}$ & & $\begin{array}{c}61 \\
(27.5 \%)\end{array}$ & $\begin{array}{c}142 \\
(25.1 \%)\end{array}$ & \\
\hline & $\begin{array}{c}\text { Inadequate } \\
\text { or fewer }\end{array}$ & $\begin{array}{c}141 \\
(38.1 \%)\end{array}$ & $\begin{array}{c}27 \\
(36.5 \%)\end{array}$ & $\begin{array}{c}111 \\
(36.0 \%)\end{array}$ & $\begin{array}{c}10 \\
(27.8 \%)\end{array}$ & $<0.001$ & $\begin{array}{c}183 \\
(39.0 \%)\end{array}$ & $\begin{array}{c}106 \\
(33.2 \%)\end{array}$ & 0.202 & $\begin{array}{c}88 \\
(39.6 \%)\end{array}$ & $\begin{array}{c}201 \\
(35.5 \%)\end{array}$ & 0.235 \\
\hline & No & $\begin{array}{c}161 \\
(43.5 \%)\end{array}$ & $\begin{array}{c}27 \\
(36.5 \%)\end{array}$ & $\begin{array}{c}93 \\
(30.2 \%)\end{array}$ & $\begin{array}{c}15 \\
(41.7 \%)\end{array}$ & & $\begin{array}{c}166 \\
(35.4 \%)\end{array}$ & $\begin{array}{c}130 \\
(40.8 \%)\end{array}$ & & $\begin{array}{c}73 \\
(32.9 \%)\end{array}$ & $\begin{array}{c}223 \\
(39.4 \%)\end{array}$ & \\
\hline \multirow{3}{*}{$\begin{array}{l}\text { Are you and your } \\
\text { colleagues and staff } \\
\text { well trained in } \\
\text { infection control at } \\
\text { your clinic or } \\
\text { workplace? }\end{array}$} & Yes & $\begin{array}{c}165 \\
(44.6 \%)\end{array}$ & $\begin{array}{c}58 \\
(78.4 \%)\end{array}$ & $\begin{array}{c}168 \\
(54.5 \%)\end{array}$ & $\begin{array}{c}17 \\
(47.2 \%)\end{array}$ & & $\begin{array}{c}237 \\
(50.5 \%)\end{array}$ & $\begin{array}{c}171 \\
(53.6 \%)\end{array}$ & & $\begin{array}{c}95 \\
(42.8 \%)\end{array}$ & $\begin{array}{c}313 \\
(55.3 \%)\end{array}$ & \\
\hline & $\begin{array}{c}\text { Inadequate } \\
\text { or fewer }\end{array}$ & $\begin{array}{c}148 \\
(40.0 \%)\end{array}$ & $\begin{array}{c}13 \\
(17.6 \%)\end{array}$ & $\begin{array}{c}96 \\
(31.2 \%)\end{array}$ & $\begin{array}{c}14 \\
(38.9 \%)\end{array}$ & $<0.001$ & $\begin{array}{c}164 \\
(35.0 \%)\end{array}$ & $\begin{array}{c}107 \\
(33.5 \%)\end{array}$ & 0.660 & $\begin{array}{c}91 \\
(41.0 \%)\end{array}$ & $\begin{array}{c}180 \\
(31.8 \%)\end{array}$ & 0.007 \\
\hline & No & $\begin{array}{c}57 \\
(15.4 \%)\end{array}$ & $\begin{array}{c}3 \\
(4.1 \%)\end{array}$ & $\begin{array}{c}44 \\
(14.3 \%)\end{array}$ & $\begin{array}{c}5 \\
(13.9 \%)\end{array}$ & & $\begin{array}{c}68 \\
(14.5 \%)\end{array}$ & $\begin{array}{c}41 \\
(12.9 \%)\end{array}$ & & $\begin{array}{c}36 \\
(16.2 \%)\end{array}$ & $\begin{array}{c}73 \\
(12.9 \%)\end{array}$ & \\
\hline $\begin{array}{l}\text { Does your clinic have } \\
\text { adequate infection }\end{array}$ & Yes & $\begin{array}{c}131 \\
(35.4 \%)\end{array}$ & $\begin{array}{c}52 \\
(70.3 \%)\end{array}$ & $\begin{array}{c}201 \\
(65.3 \%)\end{array}$ & $\begin{array}{c}19 \\
(52.8 \%)\end{array}$ & 0.001 & $\begin{array}{c}242 \\
(51.6 \%)\end{array}$ & $\begin{array}{c}161 \\
(50.5 \%)\end{array}$ & 0.092 & $\begin{array}{c}105 \\
(47.3 \%)\end{array}$ & $\begin{array}{c}298 \\
(52.7 \%)\end{array}$ & 0.235 \\
\hline
\end{tabular}




\begin{tabular}{|c|c|c|c|c|c|c|c|c|c|c|c|c|}
\hline \multirow{2}{*}{$\begin{array}{l}\text { control or personal } \\
\text { protective } \\
\text { equipment and } \\
\text { material? }\end{array}$} & $\begin{array}{c}\text { Inadequate } \\
\text { or fewer }\end{array}$ & $\begin{array}{c}154 \\
(41.6 \%)\end{array}$ & $\begin{array}{c}16 \\
(21.6 \%)\end{array}$ & $\begin{array}{c}83 \\
(26.9 \%)\end{array}$ & $\begin{array}{c}11 \\
(30.6 \%)\end{array}$ & & $\begin{array}{c}146 \\
(31.1 \%)\end{array}$ & $\begin{array}{c}118 \\
(37.0 \%)\end{array}$ & & $\begin{array}{c}76 \\
(34.2 \%)\end{array}$ & $\begin{array}{c}188 \\
(33.2 \%)\end{array}$ & \\
\hline & No & $\begin{array}{c}85 \\
(23.0 \%)\end{array}$ & $\begin{array}{c}6 \\
(8.1 \%)\end{array}$ & $\begin{array}{c}24 \\
(7.8 \%)\end{array}$ & $\begin{array}{c}6 \\
(16.7 \%)\end{array}$ & & $\begin{array}{c}81 \\
(17.3 \%)\end{array}$ & $\begin{array}{c}40 \\
(12.5 \%)\end{array}$ & & $\begin{array}{c}41 \\
(18.5 \%)\end{array}$ & $\begin{array}{c}80 \\
(14.1 \%)\end{array}$ & \\
\hline \multirow{3}{*}{$\begin{array}{l}\text { Do you have clear } \\
\text { guidelines from } \\
\text { Hospital/ health } \\
\text { department for } \\
\text { infection prevention } \\
\text { at work? }\end{array}$} & Yes & $\begin{array}{c}218 \\
(58.9 \%)\end{array}$ & $\begin{array}{c}60 \\
(81.1 \%)\end{array}$ & $\begin{array}{c}226 \\
(73.4 \%)\end{array}$ & $\begin{array}{c}25 \\
(69.4 \%)\end{array}$ & \multirow{3}{*}{$<0.001$} & $\begin{array}{c}319 \\
(68.0 \%)\end{array}$ & $\begin{array}{c}210 \\
(65.8 \%)\end{array}$ & \multirow{3}{*}{0.726} & $\begin{array}{c}140 \\
(63.1 \%)\end{array}$ & $\begin{array}{c}389 \\
(68.7 \%)\end{array}$ & \multirow{3}{*}{0.314} \\
\hline & $\begin{array}{c}\text { Inadequate } \\
\text { or fewer }\end{array}$ & $\begin{array}{c}95 \\
(25.7 \%)\end{array}$ & $\begin{array}{c}9 \\
(12.2 \%)\end{array}$ & $\begin{array}{c}61 \\
(19.8 \%)\end{array}$ & $\begin{array}{c}9 \\
(25.0 \%)\end{array}$ & & $\begin{array}{c}99 \\
(21.1 \%)\end{array}$ & $\begin{array}{c}75 \\
(23.5 \%)\end{array}$ & & $\begin{array}{c}55 \\
(24.8 \%)\end{array}$ & $\begin{array}{c}119 \\
(21.0 \%)\end{array}$ & \\
\hline & No & $\begin{array}{c}57 \\
(15.4 \%)\end{array}$ & $\begin{array}{c}5 \\
(6.8 \%)\end{array}$ & $\begin{array}{c}21 \\
(6.8 \%)\end{array}$ & $\begin{array}{c}2 \\
(5.6 \%)\end{array}$ & & $\begin{array}{c}51 \\
(10.9 \%)\end{array}$ & $\begin{array}{c}34 \\
(10.7 \%)\end{array}$ & & $\begin{array}{c}27 \\
(12.2 \%)\end{array}$ & $\begin{array}{c}58 \\
(10.2 \%)\end{array}$ & \\
\hline \multirow{3}{*}{$\begin{array}{l}\text { Have you been } \\
\text { compensated for } \\
\text { reduced working } \\
\text { hours during } \\
\text { outbreaks? }\end{array}$} & Yes & $\begin{array}{c}132 \\
(35.7 \%)\end{array}$ & $\begin{array}{c}40 \\
(54.1 \%)\end{array}$ & $\begin{array}{c}151 \\
(49.0 \%)\end{array}$ & $\begin{array}{c}11 \\
(30.6 \%)\end{array}$ & & $\begin{array}{c}198 \\
(42.2 \%)\end{array}$ & $\begin{array}{c}136 \\
(42.6 \%)\end{array}$ & & $\begin{array}{c}87 \\
(39.2 \%)\end{array}$ & $\begin{array}{c}247 \\
(43.6 \%)\end{array}$ & \multirow{3}{*}{0.134} \\
\hline & $\begin{array}{c}\text { Inadequate } \\
\text { or fewer }\end{array}$ & $\begin{array}{c}65 \\
(17.6 \%)\end{array}$ & $\begin{array}{c}12 \\
(16.2 \%)\end{array}$ & $\begin{array}{c}53 \\
(17.2 \%)\end{array}$ & $\begin{array}{c}7 \\
(19.4 \%)\end{array}$ & 0.002 & $\begin{array}{c}88 \\
(18.8 \%)\end{array}$ & $\begin{array}{c}49 \\
(15.4 \%)\end{array}$ & 0.429 & $\begin{array}{c}48 \\
(21.6 \%)\end{array}$ & $\begin{array}{c}89 \\
(15.7 \%)\end{array}$ & \\
\hline & No & $\begin{array}{c}173 \\
(46.8 \%)\end{array}$ & $\begin{array}{c}22 \\
(29.7 \%)\end{array}$ & $\begin{array}{c}104 \\
(33.8 \%)\end{array}$ & $\begin{array}{c}18 \\
(50.0 \%)\end{array}$ & & $\begin{array}{c}183 \\
(39.0 \%)\end{array}$ & $\begin{array}{c}134 \\
(42.0 \%)\end{array}$ & & $\begin{array}{c}87 \\
(39.2 \%)\end{array}$ & $\begin{array}{c}230 \\
(40.6 \%)\end{array}$ & \\
\hline
\end{tabular}

\section{Differences based on geographical location}

Except for respondents from Malaysia and GCC, the health professionals from other countries ( $>40 \%$ respondents) reported that they have not received any specialized training on containing contagious diseases. Approximately 78.4\% of the health care professionals in Malaysia felt that they were well trained in infection control practices, while the corresponding figure from India was $44.6 \%$. The availability of personal protective equipment and materials in their workplace was reported to be highest among the respondents from Malaysia (70.3\%) and least in India (35.4\%). Regarding the compensation for reduced working hours during outbreaks, participants from Malaysia reported high percentage of compensation received (54.1\%) followed by the GCC countries $(49.0 \%)$, whereas, participants from India (46.8\%) reported that they were not compensated.

\section{Differences based on profession and age}

There were no significant differences observed between age groups and between the different professions in the domain of preparedness during pandemic where the $p$ values were $>0.09$.

\section{Discussion}

Like natural catastrophes and incidents of mass violence, pandemics increase levels of fear and result in avoidance behaviors (13). Preparedness for pandemics has come under scrutiny in recent years. Whether epidemic or pandemic, healthcare workers are at the forefront of every given single outbreak and in accordance with their obligation, risk their lives. Such health workers are not only under tremendous stress during the pandemic but can also experience long-term psychological consequences post-pandemically. Thus, the problems exist within health care sectors for detecting potential pandemic emergencies at the earliest possible point and further practice. This became clear with the SARS epidemic, and most recently with the outbreak of the Ebola virus (14). No two outbreaks of disease are the same, and each has its own unique impact on health workers facing the disease $(14,15)$.

\section{Fear of neglect and ostracism}

Millions of healthcare workers providing medical care to patients are at significantly increased risk of being infected with COVID-19. An added challenge is this worry, which includes the fear of an increased risk of infection and the uncertainty that they may carry COVID-19 coronavirus home and infect their loved ones. Social ostracism is another stimulus for the increasing emotional breakdown of HCPs especially in developing Asian countries like India. Many deterring reports of the public coldshouldering HCPs have been published in social media and local newspapers $(16,17)$. House owners and innkeepers to vacate their residences without prior notice have threatened doctors and nurses. Many workers were not allowed to utilize public transport facilities and their children were ostracized (18). Following these tragic incidents, the International Federation of the Red Cross in association with other 
humanitarian associations issued a declaration against harassment, stigmatization and physical assault of HCPs involved in the COVID-19 battle (18).

Fear of disseminating disease and Need for a strategical healthcare system

Soaring mental and emotional perturbations were often reported among clinicians and other HCPs. Healthcare workers will become infected in large numbers early in the pandemic and will need to take care of themselves first. Across countries, it was found that $43 \%$ of the participants in the current research felt inadequate workforce to handle the increased demand. Other reports (19) also reflect on the mental and psychological overload of health workers; their work-related concerns and possible methods of restoration. -

Although, ethically, maximum health care professionals (86.5\%) were willing to provide medical care during the COVID-19 pandemic, about 46\% of HCPs acknowledged the risk of contracting the virus as a result of their profession. Among other previous researches, this result of our survey was similar, showing various groups of HCPs voicing a professional responsibility to provide health care during the pandemic $(20,21)$. As the pandemic takes a toll on the emotional well-being of HCPs, it is high time to implement recuperation strategies to alleviate the stress and fear. Flexibility in working hours, assigning sterilized exclusive resting rooms, special supporting teams to invigorate mental health and healthy recreation facilities during relaxing hours are some life changing approaches to combat stress (22). Similarly, the fear of contracting infection can be combated by taking diligent measures such as wearing adequate Personal Protective Equipment (PPE), no shaking hands with others, minimizing socialization and maintaining proper physical distance with fellow colleagues.

\section{Preparedness}

In response to the COVID-19 pandemic, as of April 15, 2020, the countries that participated in this research had crossed the second peak of the pandemic. At this juncture understanding the psychological and stress impact of the COVID-19 outbreak among health care workers is crucial in guiding policies and interventions to maintain their psychological well-being. It was observed that the participants from GCC exhibited more work-related stress than their counterparts. This can be attributed to inadequate manpower, contrasting to a previous commentary which proclaimed that the Kingdom of Saudi Arabia has one of the best health care systems in the GCC (23).

In terms of training and availability of proper equipment, the professionals from India felt inadequately equipped to face the pandemic. The more rational justification for this point would be that India is overpopulated compared to other countries participating in the study. Other reasons need to be further investigated to support this statement. One encouraging finding was that most of Malaysia $(70.3 \%)$ and the Gulf Co-operation countries' (65.3\%) health care workers were confident that their employers or in turn the Ministry of Health of respective countries had their medical needs taken care of. This positivity displayed by the respondents may be attributed in part to the proactive care exhibited by their respective ministries to health care workers who contracted infections in the past outbreaks. We deem this as a crucial factor in supporting and encouraging health care workers.

In comparison between the medical and dental professionals for the preparedness of the pandemic, the statistics established an equal response rate from both the groups in terms of specialized training and have received a clear guideline from health department for infection prevention at work. More work stress was reported in the medical fraternity than their dental counterparts. The hypothetical reason for this state of mind could be that during COVID-19 pandemic, the regular workload of the dental fraternity was significantly less and they were now volunteering to a new phase of work (COVID-19 duties).

Dentists and dental specialists maintain high academic standards equivalent to any medical professional programs, during which they undergo rigorous training to achieve a dental degree. With structured short-term training, the trained dentists will be talented to contribute to the larger public health domain in any such pandemic outbreaks.

\section{Limitations of the study}

We were able to contact as many medical and dental professionals as possible; many of the professionals were not able to complete the study as they had busy COVID-19 ward duties. We observed incomplete responses, as the questionnaire was quite lengthy and exhausting. Also, similar surveys were emanating in the internet from diverse sources $(24,25)$. So the response was limited. Another limitation of the study is that the female $(55.5 \%)$ respondents were comparatively more than the male $(44.5 \%)$ respondents. This can give way to gender based results on emotional and psychological elements. We 
chose to gauge the study based on age, country and profession rather than gender. The study was conducted at a time when fear of the pandemic was rampant across cultures.

\section{Conclusion}

The pandemic COVID-19 threat is significant and overwhelming, with expected levels of clinical attack and morbidity that could cause major social and economic damage, putting tremendous pressure on an already lingering health care system. The response of workers from all health care sectors to this stress will decide how well the health service will cope in the course of this crisis. Careful management of these factors will make it possible to implement strategies that address the concerns and fears of healthcare workers and remove potential barriers to work. The emotional wellbeing of these HCPs should be considered as a mandatory criterion as they confront the dread of the disease directly. A mentally and emotionally strong medical workforce is the need of the hour.

\section{Resumo}

0 novo Coronavirus 2019 (COVID-19) tem causado sérias repercussões tanto fisicas como mentais. A crise colocou uma enorme carga de trabalho sobre a comunidade global de saúde. 0 objetivo foi estudar o estado emocional e psicológico da comunidade médica e dentária em sete paises asiáticos (Índia, Malásia, os paises da Cooperação do Golfo (GCC) e, outros) e também deduzir o grau de preparo mental à medida que conduzem a guerra contra a COVID-19. Foi realizado um estudo transversal e descritivo online entre potenciais participantes de fóruns online e outras comunidades sociais relacionadas com a saúde. Foi aplicada uma análise bivariada com estatística descritiva para decifrar os resultados. Foram analisadas um total de 788 respostas completas. A taxa de resposta foi de $77,1 \%$. Os resultados revelaram a percepção dos Profissionais de Saúde sobre a COVID-19 e as suas implicações na sua vida pessoal e profissional. Foram analisadas muitas categorias do ponto de vista psicológico e emocional. Os HCP mais velhos (acima de 35 anos) relataram niveis elevados de stress no local de trabalho $(p=0,002)$. Cerca de $43,5 \%$ dos HCP da Índia relataram não ter recebido qualquer formação especializada sobre a contenção de COVID-19 ou qualquer doença contagiosa. 0 estresse emocional intenso foi relatado pelos HCPs quando os colegas foram infectados. Os profissionais médicos $(61,7 \%)$ exibiram mais stress de trabalho em comparação com os seus homólogos dentários. A análise do estado psicológico e emocional dos HCP é imperativa, especialmente nesta situação de COVID-19. Análises semelhantes são cruciais para avaliar a qualidade do nosso sistema de saúde e tomar as ações necessárias como a formação da força de trabalho, a renovação das infra-estruturas, e a regulação do fluxo de trabalho.

\section{References}

1. Godkin D, Markwell H. The duty to care of healthcare professionals: ethical issues and guidelines for policy development. SARS Expert Panel Secretariat 2003; 9-13.

2. Sokol DK. Virulent epidemics and scope of healthcare workers' duty of care. Emerg Infect Dis 2006;12(8): 1238-1241.

3. Cheung T, Fong TK, Bressington D. COVID-19 under the SARS Cloud: Mental Health Nursing during the Pandemic in Hong Kong. J Psychiatr Ment Health Nurs 2020.

4. $X u$ X, Peng $X$, Li $Y$, Cheng L, Zhou $X$, Ren B. Transmission routes of 2019-nCoV and controls in dental practice. Int J Oral Sci 2020;12(1): 1-9.

5. Pappa $S$, Ntella V, Giannakas T, Giannakoulis VG, Papoutsi $E_{1}$ Katsaounou P. Prevalence of depression, anxiety, and insomnia among healthcare workers during the COVID-19 pandemic: A systematic review and meta-analysis. Brain Behav Immun 2020;88:901-907.

6. Mostoufi B, Ashkenazie Z, Abdi J, Chen E, DePaola LG. COVID-19 and the dental profession: Establishing a safe dental practice for the coronavirus era. J Global Oral Health 2020;3(1):41.

7. Richardson H. Coronavirus: Dentists to help staff new hospitals. BBC News. 2020. Retrieved from: https://www.bbc.com/news/education-52050506.

8. Coulthard P. Dentistry and coronavirus (COVID-19)-moral decision-making. Br Dent J 2020; 228(7):503-505.

9. Abolfotouh MA, AlQarni AA, Al-Ghamdi SM, Salam M, Al-Assiri MH, Balkhy HH. An assessment of the level of concern among hospital-based health-care workers regarding MERS outbreaks in Saudi Arabia. BMC Infect Dis 2017 Jan 3;17(1):4. 
10. Novel coronavirus disease 2019 (COVID-19) pandemic. (2020, March 12). European Centre for Disease Prevention and Control. https://www.ecdc.europa.eu/sites/default/files/documents/RRA-sixthupdate-Outbreak-of-novel-coronavirus-disease-2019-COVID-19.pdf

11. 5 healthcare workers among 48 new corona cases in Saudi Arabia. (2020, March 21). Saudi Gazette. https://saudigazette.com.sa/article/591102/SAUDI-ARABIA/5-healthcare-workers-among-48new-corona-cases-in-Saudi-Arabia.

12. Cheong SK, Wong TY, Lee HY, Fong YT, Tan BY, Koh GC, et al. Concerns and Preparedness for an Avian Influenza Pandemic: A Comparison between Community Hospital and Tertiary Hospital Healthcare Workers. Ann Acad Med Singap 2008;37:96-102.

13. Lau JT, Griffiths S, Choi KC, Tsui HY. Avoidance behaviors and negative psychological responses in the general population in the initial stage of the H1N1 pandemic in Hong Kong. BMC Infect Dis 2010;10(1):139-51.

14. Lehmann $M$, Bruenahl $C A$, Löwe $B$, Addo $M M$, Schmiedel $S$, Lohse AW, et al. Ebola and psychological stress of health care professionals. Emerg Infect Dis 2015;21:913-4.

15. Lin $\mathrm{CY}$, Peng YC, Wu YH, Chang J, Chan CH, Yang DY. The psychological effect of severe acute respiratory syndrome on emergency department staff. Emerg Med J 2007;24:12-7.

16. Aravind I. Covid-19: How healthcare workers are paying a heavy price in this battle. Economic Times 2020. https://economictimes.indiatimes.com/news/politics-and-nation/covid-19-how-healthcareworkers-are-paying-a-heavy-price-in-this-battle/articleshow/75099895.cms?from=mdr.

17. Bouchard JP. Covid-19: health care workers between heroism and ostracism. Revue de L'infirmiere 2020;69(260-261):31-32.

18. Bagcchi S. Stigma during the COVID-19 pandemic. Lancet Infect Dis 2020;20(7):782.

19. Shanafelt T, Ripp J, Trockel M. Understanding and Addressing Sources of Anxiety among Health Care Professionals during the COVID-19 Pandemic. J Am Med Assoc. 2020;323(21):2133-2134.

20. Ehrenstein BP, Hanses $F$, Salzberger B. Influenza pandemic and professional duty: family or patients first? A survey of hospital employees. BMC Public Health. 2006;6:311.

21. Hogg W, Huston P, Martin C, Soto E. Enhancing public health response to respiratory epidemics: are family physicians ready and willing to help? Can Fam Physician 2006;52:1254-60.

22. Chen M, Kang L, Ma S. Impact on mental health and perceptions of psychological care among medical and nursing staff in Wuhan during the 2019 novel coronavirus disease outbreak: A crosssectional study. Brain Behav Immun. 2020;1-7.

23. AlHumaid J, Ali S, Faroog I. The psychological effects of the COVID-19 pandemic and coping with them in Saudi Arabia. Psychol Trauma 2020;12(5): 505-507.

24. FE Bureau. Life Online: Net services on a high post-COVID. Financial Express 2020. https://www.financialexpress.com/industry/technology/life-online-net-services-on-a-high-postcovid/2087404/

25. Varshney M, Parel JT, Raizada N, Sarin SK. Initial psychological impact of COVID-19 and its correlates in Indian Community: An online (FEEL-COVID) survey. PLoS ONE 2020;15(5): e0233874.

Received: 08/07/2020

Accepted: 22/03/2021 